\section{(10)rininal Artirlep.}

\section{TRICHINOSIS.*}

BY ThOMAS F. LEEN, M.D., BOSTON.

Physician in Chief to the Carney Hospital, and Assistant in the Theory and Practice of Physic at the Harvard Medical School.

OF parasitic diseases none better than trichinosis opened up pathways for scientific progress and study, from the time in 1828 when small calcareous bodies were noticed in human muscle, up to 1898 , when Thayer and Brown found practically pathognomonic blood changes pointing to an animal parasite in this disease. And at present, though of great economic interest, it has thrown open to biologists important questions, one in particular, the origin of the eosinophile, which, if discovered will be an important scientific acquisition. Clinically, the disease is a definite entity, with a symptom-complex based on pathological soundness, and though not frequent may be generally readily diagnosed. From this view it will be presented, as well as its interesting medical history, and the simple pathology of the parasite, which in every disease is of the utmost importance for proper understanding and treatment.

During the past six years there have been two cases at the Carney Hospital, the more recent one last October.

It is a febrile infectious disease appearing sporadically or epidemically, originating by infection with an animal parasite, a round worm, the trichinella spiralis, contained in pork, eaten raw or insufficiently cooked. Before 1860 , the presence of the parasites in the muscles was regarded as an anatomical curiosity, until Zenker reported clinically his classical case in Virchuw's Archives, Band xviii, which may be said to be the most important helminthological contribution of the 19th century.

Case 1. A girl aged 20 was admitted to the Dresden City Hospital on Jan. 12, 1860. She had been ailing from Christmas and about New Year had to go to bed, complaining of debility, sleeplessness, loss of appetite, constipation, fever and thirst. At first the fever was very intense and the abdomen distended and painful. Although there was no enlargement of the spleen, and rose-spots were absent, the diagnosis of typhoid was made. Very soon there developed throughout the entire muscular system, extreme pain, particularly in the extremities, so that she complained day and night. The arms and legs were bent at the elbows and knee-joints, and could not be extended without the greatest pain. An edematous swelling of the legs was present. Later a low typhoidal pneumonia appeared. The patient became profoundly apathetic and died on Jan. 27. The autopsy was performed next day, and to quote Zenker, "one can judge of my astonishment when $I$, in the first microscopic preparation, saw at a glance a dozen, non-encapsulated free trichinae in the parenchyma of the muscles." Further investigation showed the muscles

* Delivered Dec. 9, 1912, before the South Boston Medical Society. swarming with worms. No involvement of Peyer's patches was present.

In this case he determined two points of vital importance, first, the presence of living adult trichinae in the intestines, and, second, found muscle-trichinae in the flesh of the animal, a portion of which the girl had eaten. To this newly discovered disease Zenker gave the name Trichiniasis. At once the disease began to be recognized, especially in Germany, where for years past inexplainable epidemics caused large mortality, supposed to be due to a chemical poison from rotten meat.

The first great epidemic of etiological importance occurred in Saxony in 1863, with a mortality of 16 per cent. Great astonishment was caused throughout Germany in 1865, when in the town of Hedersleben of 2,000 inhabitants, 337 were infected and 101 died. Klopsch in 1866 amputated a patient's breast for carcinoma, and while operating, found in the pectoral muscle encapsulated trichinellae which were still living. The affected patient remembered that in 1842, twenty-four years before, she, with two of her family had been taken sick for several weeks with violent bone, joint and muscle pain, and edema.

In 1851, several people near Hamburg, after eating ham, were taken sick, and three died. A cat having eaten also of it began to move about very slowly and in evident pain, later dying. The cause of the disease was unknown. One of those affected became insane, and at his death in 1865, Tungel sent a piece of his muscle to Virchow, who found it almost entirely infiltrated with calcareous trichinella capsules. Freed from the capsules the embryos gave positive signs of life and infected rabbits fed on them. This shows an exceptionally long life and ability of the encapsulated forms to infect for thirteen and a half years.

Geographically, it is universal, and epidemics have been reported in Germany, England, Austria, Switzerland, France, Russia ; and in North America, first found in swine by Leidy in 1846 and in humans in 1864. It is widespread in North American swine.

Etiology. As Zenker first showed, the disease in humans is due to the eating of trichinous pork, and that its habitat is in swine. As well the rat is a carrier as it spontaneously infects. itself. They have been found also in the wild. boar, mouse, cat, dog and some others. Experimentally the infection was produced in herbivora,-rabbits and guinea-pigs. The hog for man is always the most deadly carrier of this dangerous parasite. An infection by beef, veal, mutton or horse-flesh has never been observed. The trichinella spiralis is of the class of roundworms, parasitic in man and different animals, living sexually separated, and when isolated visible to the eye. In 1835 it was named by Owen, trichina spiralis, and sex differentiation was: made out by Virchow and Leukart. 
The whole development-cycle combines essentially three stages: (1) the stage of sexual maturity or intestinal trichinae; (2) the emigration and growing of the young embryos in the muscle; and (3) the resting-stage or the muscle-trichinae.

The First Stage, or Intestinal Trichinae. When the infected pork is taken into the stomach the action of the gastric juice frees from their capsule, and connective tissue covering, the trichinae from the ingested muscle, and moving along into the intestine in two or three days they grow to mature males and females. Then copulation begins and also fertilization of the eggs. Four to five days after fertilization, that is, six to seven days after eating the trichinous pork, the birth of the embryos begin.

Without entering too deeply into the anatomy of the worm, there are a few facts you should know to aid you in the explanation of the migration of the embryos. The adult female is longer than the male, just over $3 \mathrm{~mm}$., and the male one-half as long. Up to the period of copulation they are about the same size, but after there is further growth of the female due to the stretching of the uterus. The caudal end of the female contains the ovary and from it the eggs pass upward through the oviduct to the uterus, where they are fertilized, and developing into embryos are extended into the vagina, which empties at the anterior fifth of the body. They appear first, as I have stated, six or seven days after the release of the muscle trichinae. At this time we find all stages of embryonic development, from eggs before fertilization, up to the perfectly formed embryo ready to be deposited by his mother.

The Second Stage of Development-the Embryos. As they are born they are very small and the sexes show no appreciable difference in size, as the adult, but are slender cylindrical worms, .08-.12 mm. Just before their birth their mothers bury themselves in the mucus and lie snake-like about the intestinal villi or penetrate the erypts of the lower duodenum and upper ileum. With a boring motion of their anterior end they raise up the epithelium and work into the stratum proprium or basement membrane of the mucous membrane, thus escaping eviction by peristalsis and moving intestinal contents empty their young brood directly into the lymph spaces. Only exceptionally are they born in the intestinal lumen, and it is then a question if they ever find their way to the lymph-spaces. They are born on an average of one every onehalf hour, and the birth of countless embryos from the same female extends over several weeks. Staubli reckons 1000 embryos to a female, and Leukart 1500, and Krabbe fed 400 muscle trichinellae to a rabbit, and five and onehalf weeks later its muscles contained several hundred thousand young worms.

How do they arrive at their destination, the striated musculature? This has been the subject of much discussion. The embryos, freed from the females in the intestinal walls, seek out, by their ability to move, the lymph vessels. Under certain conditions they are borne directly into a central lymph-vessel, and with the lymph they pass through the thoracic duct into the venous system to the heart, from where they are thrown with the blood, after going through the lesser circulation, into the peripheral arterial system. Hence they can be shown in the circulating blood at their maximum 8-25 days after infection.

The embryos leave the capillaries in the striated muscles, remaining only a very short time in the connective tissue, being found there only with great trouble, and penetrate quickly into the primitive muscle bundle, where they show their typical embryonic form. The fact that they are not found in the connective tissue, leads to the supposition that they are attracted to the muscle by some chemical affinity. Though the entire striated musculature may be infected, that of the diaphragm, intereostals, larynx, tongue and eye, is the more heavily hit, and this is not due to the fact of their easy and quick delivery to the groups of muscles lying in the vicinity of the diaphragm, but these muscles are the most active in the body and, therefore, have the richest blood supply. It is agreed unanimously that the beginning of migration to the muscles occurs on the ninth or tenth day after infection.

Inside the muscle fibre, the embryo quickly grows, due to osmotic absorption of food, and in ten to fourteen days it is about ten times its original size, and it begins in the third week after infection to roll itself spiral-like in the much swollen spindle-like muscle fibre, this process continuing for two weeks more, when it reaches its full growth.

Third, the Resting Stage. Hence, after four to six weeks from the ingestion of trichinous pork, the embryo enters the resting-stage, and then begins the formation of a capsule about it, taking three months for its completion, later becoming calcified. During its period of rapid growth important anatomical changes rake place, especially the development of a digestive tract and sex organs. Spirally rolled within their capsules they remain in a latent stage, perhaps for years, when they finally, after ingestion in man's or an animal's stomach, are freed from their capsules and their new life opened. Trichinellae, as well as in the blood and striated muscle, are found in the mesenteric lymph. glands, peritoneal cavity, pericardial cavity, pleural cavity, lungs and myocardium, but never as older or encapsulated forms, because as Graham has shown, they only have the power to grow in that particular type of muscle fibre which has a sarcolemma. Often heart-immunity is spoken of, as no one has ever found there perfect mature embryos. But biologically there is no immunity. Recent investigation shows that heart-muscle fibre in a certain sense possesses no sarcolemma, that is, no outside covering, and 
when the embryo tries to bore in, there is nothing to resist it, as the contractile muscular protoplasm is washed away, and it always remains outside the muscle-cells, and acting as a foreign body and irritant, an inflammatory process starts up about it and it is destroyed. This same result occurs in connective-tissue and fat. But in the destruction of muscle-fibres secondary fat development results and surrounds the encapsulated embryos.

The symptom-complex may be divided into three parts corresponding to the life-cycle and pathology of the worm, as has been stated. When and how and what the early symptoms may be is inconstant. General constitutional and gastro-enteric disturbances are the rule. General discomfort, anxiety, dull heavy headaches, dizziness, nausea and vomiting, choking sensations, chilliness, and diarrhea, with more or less violent colicky abdominal pains. The vomiting and diarrhea may become intense, and the latter may be followed later by obstinate constipation. The appetite usually disappears. The tongue shows nothing characteristic, often dry and more or less heavily coated. During this general period of lassitude an increasing fever appears. Occasionally very early an intense lameness of the muscles appears, in flexors, neck, and lumbar region. Kratz found this in mild as well as severe cases, and considered it the most constant early symptom, which after some days subsides. The patients when this and the diarrhea subsides, think they are convalescing and some try to work, but are compelled to go to bed. This muscle-lameness appears before the beginning of embryo-wandering, and it cannot be explained by muscle-invasion. Some say it is due to reflex action from the intestines, others a sympathetic affection, but Friedrichs ascribes it, as well as the severe general disturbances, edema of face and the high initial fever, to an intoxication from dead trichinellae, as seen in roundworm, fish tapeworm and other parasitic infections.

The most important pathognomonic symptom, quite constant and often generally the first, is the edematous swelling of the eyelids, upper and lower, and of the face. Seldom is it lacking and first appears on the seventh day. Somewhat later, from the ninth day on, there is edema of the extremities, more often the legs than the arms, and as well at times the scrotum. Renz twice saw scrotal edema without heart-failure, or albumen in the urine. The lid edema after some days disappears and may return later, but that of the legs is obstinate and persists a long time into convalescence. It is not easy to explain the early edema of the lids on account of the many views, as embryo-migration, vaso-constriction caused by an irritant, and that of the extremities is laid to heart weakness.

The future course of the disease develops symptoms which better be discussed systematically in relation to the individual organs.

The sensorium is generally undisturbed, though delirium has been noted in a few fatal cases, accompanied with violent attacks of dyspnea. A meningitic picture, a curled up body, stiff neck, and Kernig sign, has been present, but lumbar puncture has been negative, indicating meningismus only. Bothersome sleeplessness at the height of the disease is very frequent, and may be present early, though from it children do not suffer. Epigastric pain occurs in the second week and is ascribed to the migration of the young brood to the diaphragm. "A sudden feeling of pressure and constricting pain in the pit of the stomach, extending to the back, strikes the patient with syncope and collapse, cold hands and feet, small, intermittent pulse," symptoms similar to what Romberg has described as celiac neuralgia. This attack may last from six to twenty-four hours. followed by a profuse sweat. Complete skin anesthesia, lasting for a few days, has been reported, and itching, and paresthesia occasionally.

A characteristic symptom is profuse sweating in mild or severe cases, appearing early and lasting throughout. The skin reflexes show no essential change, but the patella, tendo-Achilles, and triceps reflexes are frequently lost or diminished, returning as patient improves. There is a change in the electrical reactions of the muscles.

Temperature may be absent, especially in children. However, in most cases there is more or less elevation and in severe cases to $104^{\circ}$ and more, beginning the first day and reaching its maximum generally in nine to eleven days Frequently the temperature curve runs as a typical typhoid, showing often morning remissions. If it continues indefinitely or is interrupted by new elevations, there are generally complications of the respiratory tract.

The pulse early is hardly more frequent than normal, from 80 to 90 , and is similar to a typhoid. When there is beginning respiratory trouble, there is an increase to 100-120. Rarely are there striking disturbances of heart activity, as intermittent pulse with bradycardia to 48 , and in women the development of soft murmurs.

Gastro-enteric symptoms, especially the early, have been spoken of. Also severe diarrhea, or obstinate constipation, with abdominal pains, can continue throughout. Mucus, muco-bloody and bloody stools have been described, the latter causing death. At autopsy in such cases gastric. duodenal and intestinal ulcers were found, and experimentally there seems to be a relation between the two, for in a trichinous infected cat, three round ulcers of the duodenum were found, and four times in infected rabbits hemorrhages and hemorrhagic erosions of the gastric mucous membrane were seen. These may be due to embolic obstruction of small vessel-areas by wandering embryos.

The spleen generally is not enlarged, though one observer noted it present in 38 out of $46 \mathrm{pa}$ tients, and if it is, it is due to a mixed infection, and not a peculiarity of the disease. 
Muscle-lameness, appearing as an early symptom, merges slowly to muscle-stiffness, due to embryo migration and ultimate changes resulting. This depends essentially on the intensity of the disease, that is, on the number of parasites piercing the muscle, for in mild cases and in children it may be absent. The patients feel as stiff as a poker, and the muscles are swollen, of wooden hardness, and very sensitive to pressure. Motion causes intense pain. All the muscles may be hit, yet the neck and lumbar muscles more so, but less the muscles of the extremities, particularly the flexor, so that the patient presents a typical posture as he lies motionless, holding his limbs in a moderate position of flexion, as though he had acute angular contractures of the elbows and knees. Muscle weakness of the highest degree may be present without considerable sense of pain. The muscle stiffness sometimes disappears in five days, and has lasted 112 , but generally in severe cases five or six weeks, corresponding to the pathologic changes, The muscles of mastication and swallowing may be affected and lead to trismus. The tongue is often difficult to move on account of its increased size, and the laryngeal muscles are severely attacked, speech-function suffering, hoarseness, and later aphonia and edema of the glottis.

The migration into the respiratory muscles, the diaphragm, intercostals and sterno-cleido-mastoids make respiration very difficult and painful, resulting in serious dyspnea, inactivity of the diaphragm, and serious hiccoughs. The respiratory interference and its sequelae play an essential part in the fatal cases. Sometimes the first symptoms may be swelling and pain in the muscles without temperature, and slowly developing and creeping leads to severe trichinosis. Sometimes cases of so-called rheumatism of the muscles, with "rheumatic pains" of one to ten years' standing, were correctly diagnosed by muscle excision and evidences of trichinella found.

The eyes sometimes have limitation of motion in conjunction with eye-pains, conjunctivitis of a severe nature, with well-marked subconjunctival ecchymoses appear. The eye-grounds are negative.

Very often bronchial catarrh, due to deficient expectoration on account of weakened respiratory muscles, with often broncho-pneumonia and a harassing cough, or frank pneumonia, is present.

The skin shows sudamnia from the profuse sweats, severe itching, and occasionally petechiae. Acne and furunculosis are frequent, and herpes rarely.

Slight urinary retention is frenuent and incontinence when there is great weakness. The urine is of the fever type and albumen and casts appear when the renal irritation is severe. A very strong diazo-reaction appears, much more so than is ever seen in typhoid or tuberculosis, and is a constant phenomenon.

The stools are diarrheic, pea-soup, or rice- water in character, occasionally bloody, or constipation may be present. Few cases have been reported where the embryos or parasite were found in the stools.

Sputum shows eosinophiles, and where blood has been raised, in a case of Askanazy, embryos were found.

The blood in trichinosis is not only of the greatest importance for differential diagnosis, but to the actual meaning and origin of the eosinophile it is of general scientific interest.

After infection the first stage shows a polycythemia and a polychromhemia, and later a mild anemia develops. There are no morphological changes, as poikilocytosis and blast-formation, and these cannot be produced experimentally in guinea-pigs, even to lethal dosage. The leucocytes diminished in typhoid are increased in trichinosis, and the great increase is due to the eosinophile. It is to the great credit of American medicine that this was proven, the first observations being made in four cases at the Johns Hopkins Hospital by Thayer and Brown in 1897. The severer the disease the greater the eosinophilia. Of 44 severe cases 33 gave estimations above 20 per cent. and of 16 mild cases only five. Of this group the highest estimate was 62.2 per cent., and experimentally in guinea-pigs 52 per cent.

In severe cases the clinical symptoms are so marked and distinctive that from them a positive diagnosis may be made, but the inestimable value of an eosinophilia in mild or moderate cases is of the greatest importance.

Experimentally after feeding trichinellae to guinea-pigs the following results were obtained:

1. A leucocytosis appeared in seven to thirteen days.

2. A genuine eosinophilia appeared.

3. The increase of the eosinophiles appeared earliest in eight days.

The eosinophilia appears not at the time of the worm infection as a result of poisonous materials from the capsule freed in the stomach, and absorbed in the intestine, nor as a result of chemical processes in the intestines, but stands in close relation to the migration of the embryos and their entrance into the musculature. For months and even years after recovery these cells are increased.

In mild cases the disease lasts two to three weeks, but much more often six to seven weeks, and in a large number of cases much longer. According to Stiles, Wardwell and Hasall in Germany were observed, from 1860 to 1880 , 8,491 cases with 513 deaths, 6.04 per cent.; from 1881 to $1896,6,326$ cases with 318 deaths, 5.02 per cent.

In epidemies the mortality varies from 30 per cent. to nil. Death occurs as early as the end of the second week and the majority from the third to the fifth week.

Here I want to report one. of our cases at the 
Carney which readily fits in with what you have heard.

CASE 2. She was 32 , single, a cook and lived in Roxbury.

Family History. Negative.

Past History. Excepting two minor accidents she had always been well. Habits showed excessive tea-drinking.

Previous Illness. Eight days before admission patient had an indefinite chill. The next morning her eyes were swollen, and she felt sick, but was up and about. Previously she had felt well excepting constipation for a week preceding. Seven days ago she vomited green mucus and food, and had a headache. Since then has been in bed most of the time with persistent severe headache, nausea and fever. Has no appetite. Under vigorous cathartics bowels moved freely. No swelling of legs. Has soreness in muscles of legs, neck and arms. Has been sleeping poorly. Has a history of eating boiled ham about ten days before the onset.

Physical Examination. W. D. and N. Rather alert young woman lying comfortably in bed. Lips slightly cyanotic. Skin clear. Slight puffiness under eyes. Pupils slightly irregular in outline, and react to light and distance. Ocular movements normal.

Teeth well cared for. Tongue cleạn, dark red. Throat reddened.

Neck. No spasm, nor tenderness of muscles.

Glands. None felt.

Chest. Well formed, expansion good on both sides; rather more on right.

Lungs. Negative.

Heart. Negative, except for some rapidity.

Radial Pulse. Small volume and not Dicrotic.

Abdomen. Negative. Spleen not felt. No rose spots.

Extremities. Very slight pitting of skin of both legs, as high as knees. No scars.

Reflexes. Knee-jerks lively. Achilles normal. No clonus; no Kernig.

Oct. 30. Stool liquid, yellow, no blood, no parasites or ova; negative to guiac test.

Blood Examination. Hgb. 90 per cent. Leucocytosis 15,200. Polynuclears, 60 per cent. Large mononuclears, 6 per cent. Small. Mononuclears, 4 per cent. Eosinophiles, 28 per cent. Transitionals, 2 per cent.

Nov. 1. Blood and 3 per cent. acetic acid in equal parts showed no parasites.

Nov. 3. Muscular tenderness has not increased.

Nov. 4. Distressing pain on breathing with cough and signs of a pleuritis in lower right back and a bronchitis found.

Nov. 5. Patient very sick, right face flushed. She is nauseated and vomits. No definite consolidation made out. Leucocytosis, 22,800. Polynuclears, 70 per cent. Eosinophiles, 4 per cent. Large mononuclears, 12 per cent. Small mononuclears, 11 per cent. Transitionals, 4 per cent. Distressing chest pains and cough severe. Urine shows blood and coarse granular casts, and albumen.

Nov. 10. Feels much better, but still has pains and cough. Temperature normal, and patient spends more comfortable nights.

Nov. 12. Leucocytosis was 18,400, and eosinophiles 7 per cent. Urine still shows irritative elements.

Nov. 15. Pains in mouth, teeth and gums, but dentist cannot find anything the trouble with teeth.
Nov. 19. Leucocytes, 12,800. Eosinophiles, 3 per cent.

Nov. 22. Very much better, no pains nor aches. Complains of much distress after meals. General improvement. was steady and she was discharged Nov. 28, 1910, thirty days after admission.

That case, you see, is typical and though it appeared in the typhoid season, one familiar with the clinical aspects of trichinosis could readily diagnose it.

As regards immunity, there is none, for every animal susceptible to it may be reinfected.

Differential Diagnosis. It is an acute febrile sickness with more or less marked gastro-enteric symptoms, lameness of the muscles, edematous swelling of the face, mainly the lids, redness, and, eventually, ecchymoses of the conjunctiva, disturbing sleeplessness, profuse sweats, muscle pains, muscle stiffness and hardness, absence of patellar and Achilles reflexes, Kernig phenomenon, absence of enlargement of the spleen, strong diazo-reaction, and blood showing leucocytosis, eosinophilia, embryos circulating in the blood, and muscle trichinae in striated muscle.

To differentiate it from typhoid is confusing because of the slow onset, gastro-enteric disturbances, a continued and ascending temperature, the apathy, the positive diazo, especially when there is no enlargement of spleen and no Widal. But against it are the face and lid edema, the marked muscle symptoms, and the later absent spleen, absence of rose-spots, and the leucocytosis and eosinophilia.

Heart and kidneys as causes of the edema may readily be excluded by stethoscope and urine; as well as inflammatory process of face or scalp.

Against sausage and meat poisoning of a toxic nature, trichinosis is not so sudden or violent, and the absence of edema of lids, muscle lameness and of blood picture.

Rheumatic troubles may be ruled out by edema of face, eosinophilia, and cause of the disease.

Acute primary polymyocitis is difficult to differentiate, as the eosinophilia does not aid, but examination of muscle from the deltoid or bieeps will show trichinellae, and perhaps the blood embryos.

Multiple neuritis is easily differentiated.

Therapy. If only a short time has elapsed after eating the infected food, copious stomach washings and thorough catharsis should be tried. Strong antithelminties, such as santonin, extract of male fern, glycerine and benzol in frequent large doses. Later the fact that the worms are pressed in the crypts and intestinal mucus, strong drugs might tend to drive the worms further into the mucous membrane. So catharties are given, as ealomel, compound infus of senna, castor oil, etc., to keep the intestine thoroughly clean and wash away any worms which have temporarily lost their grip. For direct action on the worm, glycerine, benzol, the 
picronitrate of potash and soda. Now that Ehrlich in chemico-therapeutic experiments has given us salvarsan and neosalvarsan, they are well worthy of trial to kill the embryos in the blood. Colloidal silver (kollargol) 4-6 c.c. of a 2 per cent. solution has been tried intravenously for the embryos. Symptomatic treatment, as each case demands, should be given, and diet accordingly.

Prophylaxis. The mode of infection is well known, therefore we must prevent the eating of raw or insufficiently prepared pork products, and further efforts to fight the infection in swine. All slaughtered hogs should be inspected with the microscope, for early trichinous pork cannot be detected with the naked eye, though the older forms may be seen as white calcified pinhead specks on and in the muscle. Inspection of the diaphragm, ribs, larynx, and tongue muscles should be made always, on account of their frequency of infection. No land is free of the disease. The largest percentage of infection in swine is in the United States, from 2.7 to 16.3 per cent. Curiously, we have no inspection for the pork we raise and eat, but what is exported we must inspect, especially if we wish to send it to Germany. Another factor is the rat infesting slaughterhouses and piggeries, and spreading the disease in swine. They are carnivorous and eat infected pork, and at their death are eaten by the swine. In Saxony, of 704 rats examined 8.3 per cent. were trichinous. Swine eat pork also and may be infected so.

Much work has been done scientifically on trichinosis which has not fallen into the hands of the general man, and what I have tried to give you are facts and some hypotheses about a simple disease, but one to practitioners and students that seems shrouded in difficulty and mystery.

\section{LITERATURE.}

Virchow's Archives, 1866, Band 35.

Virchow's Archives, 1863, Band 28.

Staubli: Trichinosis.

Leukart, R.: Untersuchungen über Trichina Spiralis.

Ibidem: Der geschlechtsreife Zustand der Trichina Spiralis.

Ibid.: Das Benzinals Gegenmittel gegen die Trichinen, Virchow, Archiv., 1864, Band 28.

Kratz: Die Trichienepdiemie zu Hedersleben.

Meyer, Karl: Die Klinische Bedeutung der Eosinophile, Berlin, 1905.

Merkel, G. : Behandlung der Trichinenkrankheit. Handb. d. spez. Therap. innere Krankh.

Romberg, E.: Utber die Erkrankungen des Herzmuskels bei Typhus Abdominalis, Scharlach, und Diptherie, Deutsches Archiv. für Klin. Med., Band 48, 1891.

Thayer, W. S.: On the Increase of the Eosinophile Cells, etc. The Lancet, Sept. 25, 1897.

Williams, Herbert U.: The Frequency of Trichinosis in the United States. Journal of Medical Research, Vol vi, 1901.

Osler, William: Practice of Medicine.

Strumpell, A.: Lehrbuch der speziellen Pathologie und Therapie der Inneren Krankheiten, 1912.
THE DIAGNOSTIC SIGNIFICANCE OF THE LEUCOCYTE COUNT IN OSTEOMYELITIS AND TUBERCULOSIS OF THE BONES IN CHILDHOOD.

\author{
BY EBEN W. FISKE, A.M., M.D., \\ House Surgeon; Children's Hospital, Boston.
}

(From the Orthopedic Department of the Children's Hospital,

Leucocytosis is the presence in the blood of an increased number of white corpuscles, of the same varieties as occur in normal blood. It is present in many physiological conditions, but it is of especial interest as a pathological phenomenon, taking a leading part in the reaction of the individual to infection by pyogenic bacteria. In the adult, a number exceeding 9,000 per cubic millimeter may be considered a leucocytosis, but in children, whose physiological equilibrium is normally unstable, any number of leucocytes between 6,000 and 12,000 may be considered normal.

As to the significance of leucocytosis in pyogenic and tuberculous bone infections, especially in childhood, I have been unable to find satisfactory comment in the literature, beyond the general statement that leucocytosis occurs, and is usually very high, in the former and that it is low in the latter, constituting, therefore, a basis for differential diagnosis between the two. The data, and conclusions therefrom, presented in this paper, are consequently drawn from leucocyte counts made during the last few years on patients in The Boston Children's Hospital, without reference to other findings.

Since Sept. 1, 1907, 35 children with osteomyelitis have had leucocyte counts, a total of 51 counts having been made on these cases. In the last two years, a more routine and thorough examination of patients with this disease has been made, and the average leucocyte count has been found to be lower, which may be explained by the fact that in the less recent cases, white blood counts were made only in the presence of serious symptoms, poor general condition or obscure local conditions. This count arranged by periods, is as follows :

Sept. 1, 1907 to Jan. 1, 1909, average count

Jan. 1, 1909 to Jan. 1, 1911, a verage count

Jan. 1, 1911 to present date; average count

25,800

18,300

In the last year only, the average count is 16,200

If leucocytosis of any significance be considered a leucocyte count of 12,000 and above (in patients below the age of 12), and a count of 18,000 and over signifies a distinct reaction to infection, the 51 counts taken on these cases group themselves thus:

$\begin{array}{ll}12,000 \text { and below } & 12 \text { or } 24 \% \\ 12,100 \text { to } 18,000 & 19 \text { or } 37 \% \\ 18,000 \text { and over } & 20 \text { or } 39 \%\end{array}$

As far as could be ascertained, 34 of these counts were taken in an acute stage of infection (for the most part recent in onset), 17 in chronic 